Presented at the Conference Confinement V, Gargnano, Italy, September 10-14, 2002

UNITU-THEP-29/2002

FAU-TP3-02/29

\title{
KUGO-OJIMA CONFINEMENT CRITERION, ZWANZIGER-GRIBOV HORIZON CONDITION, AND INFRARED CRITICAL EXPONENTS IN LANDAU GAUGE QCD
}

\author{
R. ALKOFER, C. S. FISCHER \\ Institute for Theoretical Physics, University of Tübingen, Auf der \\ Morgenstelle 14, D-72076 Tübingen, Germany \\ AND L. VON SMEKAL \\ Institute for Theoretical Physics III, University of Erlangen-Nürnberg, \\ Staudtstr. 7, D-91058 Erlangen, Germany
}

\begin{abstract}
The Kugo-Ojima confinement criterion and its relation to the infrared behaviour of the gluon and ghost propagators in Landau gauge QCD are reviewed. The realization of this confinement criterion (which in Landau gauge relates to Zwanziger's horizon condition) results from quite general properties of the ghost DysonSchwinger equation. The numerical solutions for the gluon and ghost propagators obtained from a truncated set of Dyson-Schwinger equations provide an explicit example for the anticipated infrared behaviour. These results are in good agreement, also quantitatively, with corresponding lattice data obtained recently. The resulting running coupling approaches a fixed point in the infrared, $\alpha(0)=8.9 / N_{c}$. Solutions for the coupled system of Dyson-Schwinger equations for the quark, gluon and ghost propagators are presented. Dynamical generation of quark masses and thus spontaneous breaking of chiral symmetry is found. In the quenched approximation the quark propagator functions agree well with those of corresponding lattice calculations. For a small number of light flavours the quark, gluon and ghost propagators deviate only slightly from the quenched ones. While the positivity violation of the gluon spectral function is apparent in the gluon propagator, there are no clear indications of positivity violations in the Landau gauge quark propagator.
\end{abstract}

\section{Gluon confinement in Landau gauge and the ghost propagator}

The success of perturbative QCD for hadronic reactions at high energies provides compelling evidence that QCD is the correct theory of strong interactions and that all hadrons are made of quarks and the particles 
gluing them together, the gluons. However, we need the hypothesis of confinement in order to rescue the success of QCD. Over the last decades there have been many attempts to prove confinement from QCD. Despite these efforts it is fair to say that the phenomenon of confinement is still little understood: an undisputable mechanism responsible for this effect has not been found yet. Furthermore, we may even face the challenge that nowadays' formulation of quantum field theory is not sufficient to tackle this problem successfully: it seems not even clear, at present, whether the phenomenon of confinement is at all compatible with a description of quark and gluon correlations in terms of local fields.

There is a number of criteria which signal unambigously the occurrence of confinement. One line of research starts from the expectation that the two-point correlation functions of QCD, the quark, gluon and ghost propagators, are likely to provide some clues to the underlying structures of the theory which are responsible for confinement. And indeed, it has been argued $^{1}$ that in Faddeev-Popov quantized Landau gauge QCD the infrared behaviour of the ghost propagator is related to both, the Kugo-Ojima confinement criterium $^{2}$ and the Gribov-Zwanziger horizon condition ${ }^{3,4}$.

Kugo and $\mathrm{Ojima}^{2}$ have shown that a physical state space containing only colourless states is generated, if two conditions are satisfied: First, one should not have massless particle poles in transverse gluon correlations and, second, one needs well-defined, i.e. unbroken, global colour charges. The second condition can be related to the behaviour of the ghost propagator in Landau gauge. For it to be satisfied, the propagator must be more singular than a massless particle pole in the infrared ${ }^{1}$.

Gribov's horizon condition is connected to the gauge fixing ambiguities in the linear covariant gauge ${ }^{3}$. Ideally one would eliminate Gribov copies along gauge orbits by a restriction of the functional integral of the QCD partition function to the so-called fundamental modular region. This part of configuration space lies inside the first Gribov region, a convex region in gauge field space which contains the trivial configuration $A \equiv 0$. At the boundary of the first Gribov region, the lowest eigenvalue of the FaddeevPopov operator approaches zero. Entropy arguments have been employed to reason that the infrared modes of the gauge field are close to this Gribov horizon ${ }^{4}$. As the ghost propagator is the inverse of the Fadeev-Popov operator we therefore encounter the presence of the Gribov horizon in the infrared behaviour of the ghost: The ghost propagator is required to be more singular than a simple pole if the restriction to the Gribov region is correctly implemented. Furthermore, by the same entropy arguments, the 
gluon propagator has to vanish in the infrared ${ }^{4}$.

At this point it is interesting to note that employing Stochastic Quantiziation instead of the Faddeev-Popov formalism avoids the FaddeevPopov determinant and thus the Gribov problem completely. The ghosts being absent the above picture seems to be impossible to be realized. Nevertheless one finds essentially the same infrared behaviour for the propagator of the transverse gluons whereas the longitudinal gluons (which are absent in Faddeev-Popov-Landau gauge) take over the role of the ghosts ${ }^{5}$. Therefore the following generic picture in covariant gauges seems likely: Negative metric states like ghosts and/or longitudinal gluons are long-ranged whereas the propagator for transverse gluons vanishes for long distances.

To summarize this mechanism for gluon confinement: an infrared enhanced propagator for ghosts (or longitudinal gluons) leads to an infrared vanishing (or, at least, infrared finite) tranverse gluon propagator. As demonstrated below such a gluon propagator leads to violation of positivity in the spectral function for transverse gluons and thus describes confined transverse gluons. Speaking somewhat sloppily: In Landau gauge QCD the gluons are confined by the Faddeev-Popov ghosts which are the long-range correlations of the theory.

\section{Verifying the Kugo-Ojima Confinement Criterion}

As confinement in covariant gauges is correlated to infrared singularities we have the need for a continuum-based non-perturbative method. The framework we have chosen to investigate the behaviour of the propagators of QCD are the Dyson-Schwinger equations (DSEs) for the QCD propagators (for recent reviews see e.g. refs. ${ }^{6,7}$ ). Being complementary to lattice Monte Carlo simulations which have to deal with finite-volume effects, DSEs allow for analytical investigations of the infrared behaviour of correlation functions. In Landau gauge we have the particularly simple situation that the ghost-gluon vertex does not suffer from ultraviolet infinities. Based on this observation one can use the general structure of the ghost DSE, the properties of multiplicative renormalizability and the assumption that all involved Green's functions can be expanded in a power series to show that the KugoOjima criterion as well as the Zwanziger's horizon condition are satisfied ${ }^{8,9}$. Furthermore, it has been shown that the infrared behaviour of the ghost and the gluon propagators are uniquely related: Defining ghost and gluon renormalization functions, $Z\left(k^{2}\right)$ and $G\left(k^{2}\right)$, respectively, from the propagators $D_{\mu \nu}^{\text {Gluon }}\left(k^{2}\right)=\left(\delta_{\mu \nu}-\frac{k_{\mu} k_{\nu}}{k^{2}}\right) Z\left(k^{2}\right) / k^{2}$ and $D^{\text {Ghost }}\left(k^{2}\right)=-G\left(k^{2}\right) / k^{2}$, 
one obtains:

$$
Z\left(k^{2}\right) \sim\left(k^{2}\right)^{2 \kappa} \quad \text { and } \quad G\left(k^{2}\right) \sim\left(k^{2}\right)^{-\kappa} .
$$

The corresponding gluon propagator is thus infrared vanishing or, at least, infrared finite.

A further interesting consequence is the fact that the corresponding powers in the running coupling (as extracted from the ghost-gluon vertex) exactly cancel and one obtains an infrared fixed point for the coupling, see the next section.

\section{Propagators of Yang-Mills theory: Ghosts and Gluons}

Above we have deduced the qualitative infrared behaviour of QCD propagators applying only general principles. To obtain detailed information on the propagators of Landau gauge QCD from the DSEs they have to be truncated, and, even more severe, ansätze for the vertices have to be made. The resulting closed system of equations can be solved both, analytically in the infrared and numerically for non-vanishing momenta. The considerations presented in the previous section suggest that for small momenta the ghost loop dominates in the gluon DSE. Assuming this dominance, effects from a wide class of possible dressings for the ghost-gluon vertex have been investigated ${ }^{9}$ and found to be of negligible influence to the qualitative findings. Thus, for the purpose of this talk we concentrate on the simplest of these truncation schemes ${ }^{10,11}$. It employs a bare ghost-gluon vertex, a dressed three-gluon vertex and neglects four-gluon vertices. In addition, as confinement is expected to be present in the pure Yang-Mills sector of QCD we will couple in the quarks at a later stage.

A coupled system of gluon and ghost DSEs has been studied for the first time in ref. ${ }^{12}$. In this investigation the three-point functions have been modeled such that the Slavnov-Taylor identities have been fulfilled to high degree of accuracy. On the other hand, technical simplifications like approximating the angular integrals in the DSEs had to be employed. A study beyond Landau gauge is given in ref. ${ }^{13}$. There it is shown that the diagrams involving four-gluon vertices cannot be neglected in the analytical extraction of the infrared behaviour of the gluon and ghost propagators in the so-called Curci-Ferrari gauges if bare vertex functions are used. As a side result it has been demonstrated that in linear covariant gauges the assumption of infrared dominance of the ghost loop is, at least, self-consistent.

The truncation scheme of refs. ${ }^{10,11}$ provides the correct one-loop anomalous dimensions of the ghost and gluon dressing functions, $G\left(k^{2}\right)$ and $Z\left(k^{2}\right)$, 
respectively, and thus correctly describes the leading logarithmic behaviour of the propagators in the ultraviolet. Furthermore, this scheme reproduces the infrared exponents found in refs. ${ }^{9,14}$ :

$Z\left(k^{2}\right) \sim\left(k^{2}\right)^{2 \kappa}$ and $G\left(k^{2}\right) \sim\left(k^{2}\right)^{-\kappa} \quad$ with $\quad \kappa=(93-\sqrt{1201}) / 98 \approx 0.595$.

These exponents are close to the ones extracted from lattice calculations ${ }^{15,16,17}$. Interestingly enough they are also close to the ones obtained in a comparable truncation scheme in stochastically quantized Landau gauge Yang-Mills theory for the transverse and the longitudinal gluons ${ }^{5}$.

In Fig. 1 the numerical solutions for the gluon and ghost dressing functions for the colour group $\mathrm{SU}(2)$ are compared to those obtained from recent lattice calculations ${ }^{17}$. Differences mainly occur for the gluon dressing function in the region around its maximum, i.e. somewhat below one $\mathrm{GeV}$. These can be attributed to the omission of the two-loop diagrams in the DSE truncation. Given the limitations of both methods the qualitative and partly even quantitative agreement is remarkable.
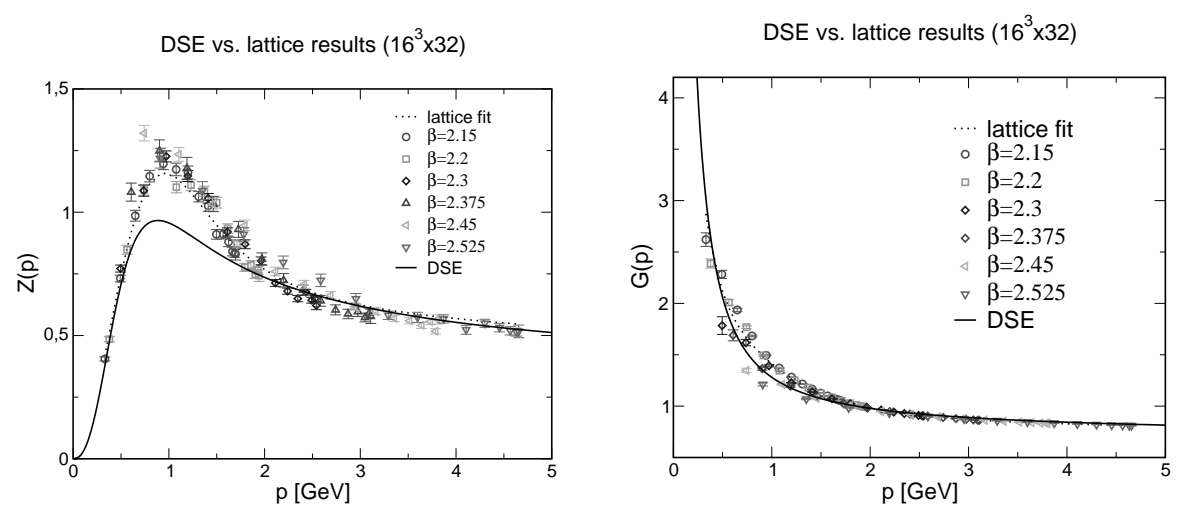

Figure 1. Solutions of the Dyson-Schwinger equations (labeled DSE) compared to recent lattice results for two colours ${ }^{17}$.

\section{Infrared behaviour of the running coupling}

The running coupling can be defined non-perturbatively as follows ${ }^{12,18}$ :

$$
\alpha\left(k^{2}\right)=\alpha\left(\mu^{2}\right) Z\left(k^{2} ; \mu^{2}\right) G^{2}\left(k^{2} ; \mu^{2}\right)
$$

where the dependence of the propagator functions on the renormalization point have been made explicit. An important point to notice in the results 
described above is the unique relation between the gluon and ghost infrared behaviour. As explained the structure of the ghost DSE and the nonrenormalization of the ghost-gluon vertex require that in Landau gauge the product $Z\left(k^{2}\right) G^{2}\left(k^{2}\right)$ goes to a constant in the infrared. The DSE result for the running coupling can be seen in Fig. 2 .

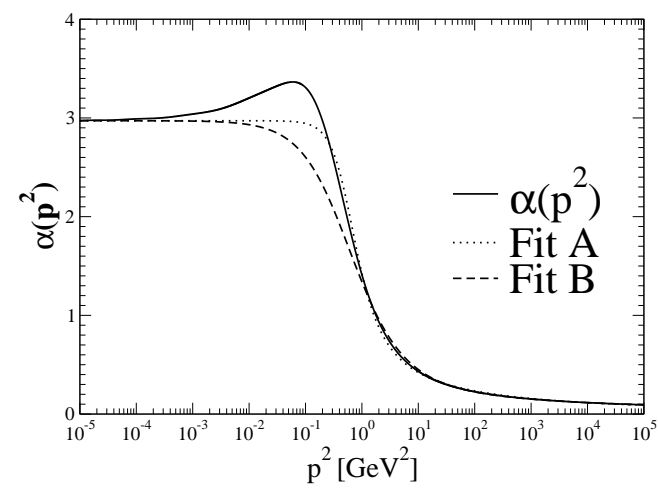

Figure 2. The strong running coupling from the DSEs and the two fits given in ref. ${ }^{18}$.

The analytically obtained value for the fixed point of the running coupling in the infrared is ${ }^{9}$

$$
\alpha(0)=\frac{4 \pi}{6 N_{c}} \frac{\Gamma(3-2 \kappa) \Gamma(3+\kappa) \Gamma(1+\kappa)}{\Gamma^{2}(2-\kappa) \Gamma(2 \kappa)} \approx 2.972
$$

for the gauge group $\mathrm{SU}(3)$ in this truncation scheme. Corrections from possible dressings for the ghost-gluon vertex have been found to be such that $2.5<\alpha(0) \leq 2.97$. The maximum at non-vanishing momenta seen in our result for the running coupling results in a multi-valued beta-function. On the other hand, it appears in a region where the above comparison to lattice data suggests that our results are least reliable. (The physical scale has been fixed by requiring the experimental value $\alpha\left(M_{Z}^{2}=(91.2 \mathrm{GeV})^{2}\right)=0.118$.) We have therefore summarized our result for the running coupling in two monotonic fit functions, see ref. ${ }^{18}$ for more details.

\section{Propagators of QCD: Ghost, Glue and Quark}

In the quark DSE as well as in the quark loop of the gluon DSE the quarkgluon vertex enters. Very recently lattice results for the quark-gluon vertex 
became available ${ }^{19}$. However, at present the statistical errors of such simulations are too still large to use the lattice results as guideline in the construction of reliable ansätze for the quark-gluon vertex. Meanwhile, we proceed with assuming that the quark-gluon vertex factorizes as follows ${ }^{20}$,

$$
\Gamma_{\nu}(q, k)=V_{\nu}^{a b e l}(p, q, k) W^{\neg a b e l}(p, q, k)
$$

with $p$ and $q$ denoting the quark momenta and $k$ the gluon momentum. Here, a non-Abelian factor $W^{\neg a b e l}$ multiplies the Abelian part $V_{\nu}^{a b e l}$, which carries the tensor structure of the vertex. For the latter we choose a construction $^{21}$ successfully used in QED, see e.g. ref. ${ }^{22}$.

The Slavnov-Taylor identity for the quark-gluon vertex implies that $W^{\neg a b e l}(p, q, k)$ has to contain factors of the ghost renormalization function $G\left(k^{2}\right)$. Due to the infrared singularity of the latter the effective low-energy quark-quark interaction is infrared enhanced as compared to the interaction generated by the exchange of an infrared suppressed gluon. Therefore the effective kernel of the quark DSE contains an (integrable) infrared singularity ${ }^{20}$. Further constraints imposed on $W^{\neg a b e l}(p, q, k)$ are such that (i) the running coupling as well as the quark mass function are, as required from general principles, independent of the renormalization point and (ii) the one-loop anomalous dimensions of all propagators are reproduced.

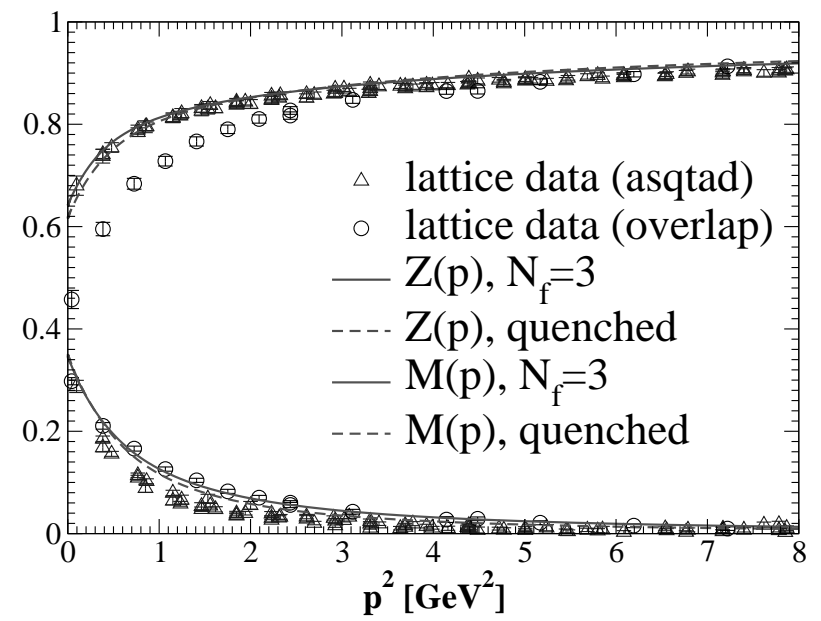

Figure 3. The quark propagator functions in quenched approximation as well as for three massless flavours compared to the lattice data of ref. ${ }^{23}$. 
In Fig. 3 we compare our results for the quark propagator $S(p)=$ $\frac{Z\left(p^{2}\right)}{i \not p+M\left(p^{2}\right)}$ in quenched approximation as well as for three massless flavours with lattice data ${ }^{23}$. As one sees the DSE results nicely agree with the one from the lattice. Furthermore, for the considered number of flavours the quenched approximation works well. (Also the gluon and the ghost functions remain almost unchanged ${ }^{20}$.)

In Fig. 4 we display the result of a possible test on positivity violations in the gluon and quark propagators for two choices of the quark-gluon vertex. Loosely speaking, negative values for the one-dimensional Fourier transforms of propagators are sufficient to demonstrate the positivity violations related to confinement. Whereas previous findings for the gluon propagator are confirmed herewith also beyond quenched approximation we have not been able to demonstrate positivity violation for the quark propagator.
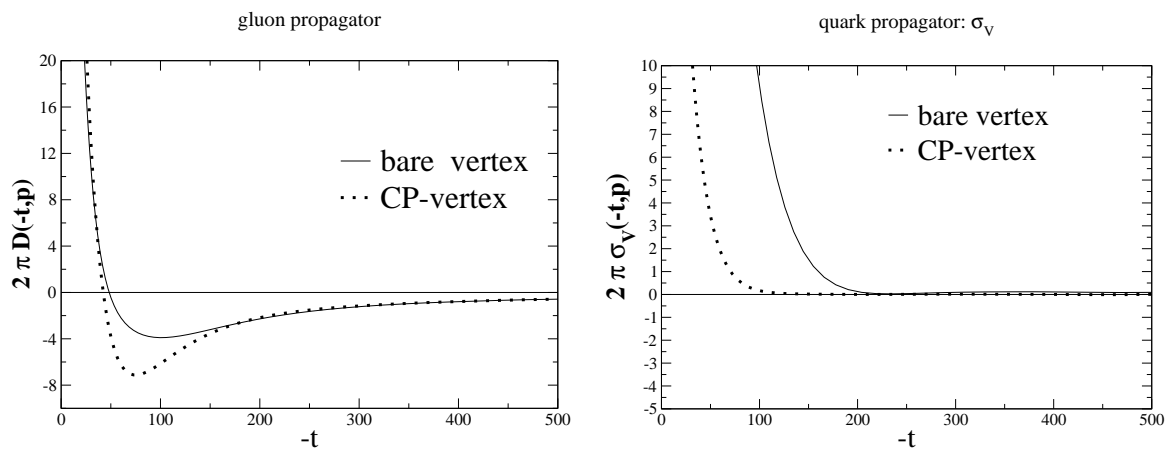

Figure 4. The one-dimensional Fourier transforms of the gluon propagator, $D\left(-t, \vec{p}^{2}\right)$, and the vector part of the quark propagator, $\sigma_{V}\left(-t, \vec{p}^{2}\right)$, are shown. We observe violation of reflection positivity for the gluon propagator but not for the quark propagator.

\section{Conclusions}

We have verified the Kugo-Ojima confinement criterion by studying the gluon, ghost and quark Dyson-Schwinger equations of Landau gauge QCD employing analytical as well numerical techniques. The resulting infrared behaviour of gluon and ghost propagators, namely a highly infrared singular ghost and an infrared suppressed gluon propagator, is related to the GribovZwanziger horizon condition. The solution for these propagators has then been used to calculate a non-perturbative running coupling for all spacelike 
momentum scales.

Dynamical chiral symmetry breaking is manifest in the obtained solutions for the quark propagator. Hereby only carefully constructed vertex ansätze have been able to generate masses in the typical phenomenological range of $300-400 \mathrm{MeV}$. The agreement with lattice data in quenched approximation confirms the quality of our truncation and in turn it shows that chiral extrapolation on the lattice works well. In the unquenched case including the quark-loop in the gluon equation with $N_{f}=3$ light quarks we obtain only small corrections compared to the quenched calculations. For a larger number of light flavours $\left(N_{f}>6\right)$ we have indications that the coupled system is changed qualitatively, and that the Kugo-Ojima confinement criterion may cease to be valid.

We tested the gluon and quark propagators for positivity violations. We confirmed previous findings that the gluon propagator shows violation of reflection positivity. We could not find similar violations for the quark propagator. With the results obtained so far we cannot exclude positivity violation for the quarks, however, one might take our result as a further indication that (in Landau gauge) the confinement mechanism for quarks differs qualitatively from the one for transverse gluons.

Studies of the gluon, ghost and quark Dyson-Schwinger equations at non-vanishing temperatures (for first results see ref. ${ }^{24}$ ) and quark densities are under way. Main goals are hereby to clarify the relation between deconfinement and chiral restauration and to possibly find the deconfinement criterion related to the Kugo-Ojima criterion.

\section{Acknowledgements}

R.A. and L.v.S. thank the Organizing Committee, and especially N. Brambilla and G. Prosperi, for their efforts to make this very nice and interesting conference possible.

We are grateful to K. Langfeld, J. M. Pawlowski, H. Reinhardt, C. D. Roberts, S. M. Schmidt, D. Shirkov, P. Watson and D. Zwanziger for helpful discussions.

This work has been supported in part by the DFG under contracts Al 279/3-4 and Sm 70/1-1 as well as by the European graduate school Tübingen-Basel (DFG contract GRK 683). 
Presented at the Conference Confinement V, Gargnano, Italy, September 10-14, 2002

\section{References}

1. T. Kugo, Int. Symp. on BRS symmetry, Kyoto, Sep. 18-22, 1995, arXiv:hepth/9511033; L. von Smekal and R. Alkofer, Proceedings of the 4th International Conference on Quark Confinement and the Hadron Spectrum, Vienna, Austria, 3-8 July 2000, arXiv:hep-ph/0009219.

2. T. Kugo and I. Ojima, Prog. Theor. Phys. Suppl. 66 (1979) 1.

3. V. N. Gribov, Nucl. Phys. B 139 (1978) 1.

4. D. Zwanziger, Nucl. Phys. B 364 (1991) 127; Nucl. Phys. B 399 (1993) 477; Nucl. Phys. B 412 (1994) 657.

5. D. Zwanziger, arXiv:hep-th/0206053.

6. R. Alkofer and L. von Smekal, Phys. Rept. 353 (2001) 281.

7. C. D. Roberts and S. M. Schmidt, Prog. Part. Nucl. Phys. 45 (2000) S1.

8. P. Watson and R. Alkofer, Phys. Rev. Lett. 86 (2001) 5239. R. Alkofer, L. von Smekal and P. Watson, Proceedings of the ECT* Meeting on Dynamical Aspects of the QCD Phase Transition, Trento, Italy, March 12-15, 2001, arXiv:hep-ph/0105142.

9. C. Lerche and L. von Smekal, Phys. Rev. D 65 (2002) 125006.

10. C. S. Fischer, R. Alkofer and H. Reinhardt, Phys. Rev. D 65 (2002) 094008.

11. C. S. Fischer and R. Alkofer, Phys. Lett. B 536 (2002) 177.

12. L. von Smekal, R. Alkofer and A. Hauck, Phys. Rev. Lett. 79 (1997) 3591; L. von Smekal, A. Hauck and R. Alkofer, Annals Phys. 267 (1998) 1.

13. R. Alkofer, C. S. Fischer, H. Reinhardt and L. von Smekal, in preparation.

14. D. Zwanziger, Phys. Rev. D 65 (2002) 094039.

15. F. D. Bonnet, P. O. Bowman, D. B. Leinweber and A. G. Williams, Phys. Rev. D 62 (2000) 051501.

16. F. D. Bonnet, P. O. Bowman, D. B. Leinweber, A. G. Williams and J. M. Zanotti, Phys. Rev. D 64 (2001) 034501.

17. K. Langfeld, H. Reinhardt and J. Gattnar, Nucl. Phys. B 621 (2002) 131. see also: K. Langfeld et al., arXiv:hep-th/0209173.

18. R. Alkofer, C. S. Fischer and L. von Smekal, Acta Phys. Slov. 52 (2002) 191. arXiv:hep-ph/0209366.

19. J. Skullerud and A. Kizilersu, JHEP 0209, 013 (2002); J. Skullerud, P. Bowman and A. Kizilersu, these proceedings, hep-lat/0212011.

20. C. S. Fischer and R. Alkofer, arXiv:hep-ph/0301094.

21. D. C. Curtis and M. R. Pennington, Phys. Rev. D 42, 4165 (1990).

22. M. R. Pennington, in Proceedings of the Workshop on Nonperturbative Methods in Quantum Field Theory, edited by A. W. Schreiber, A. G. Williams, and A. W. Thomas, p. 49, Adelaide, 1998, World Scientific; arXiv:hepth/9806200.

23. F. D. Bonnet, P. O. Bowman, D. B. Leinweber, A. G. Williams and J. B. Zhang [CSSM Lattice collaboration], Phys. Rev. D 65, 114503 (2002); J. B. Zhang, F. D. Bonnet, P. O. Bowman, D. B. Leinweber and A. G. Williams, arXiv:hep-lat/0208037; P. O. Bowman, U. M. Heller, D. B. Leinweber and A. G. Williams, arXiv:hep-lat/0209129.

24. A. Maas, B. Gruter, R. Alkofer and J. Wambach, arXiv:hep-ph/0210178. 